\title{
An audit of RCP guidelines on DMSA scanning after urinary tract infection
}

\author{
P V Deshpande, K Verrier Jones
}

\begin{abstract}
Aim-To assess the outcome of imaging investigations carried out in children with urinary tract infection (UTI), to compare the investigations with national guidelines, and to assess the impact on management.

Methods-Retrospective review of inpatients and outpatients, aged 0-12 years, referred to the University Hospital of Wales Healthcare Trust between February 1997 and January 1998 with UTI. All children without bacterial evidence of UTI and children previously investigated for antenatal urological anomalies, major congenital anomalies, or UTI were excluded.

Results-A total of 164 children (51 boys, 113 girls) were included. Thirteen of 56 infants $(23 \%)$ and $82 / 108$ older children $(76 \%)$ were diagnosed at home over one year. The prevalence of dilatation on ultrasound was $8 \%$, renal scarring on dimercaptosuccinic acid (DMSA) scan was $11 \%$, and vesicoureteric reflux (VUR) was $34 \%$ when investigations were carried out following guidelines published by the Royal College of Physicians. In children aged 1-6 years, the prevalence of scarring was $1 / 54(2 \%)$ in those treated at home and $6 / 18(33 \%)$ in inpatients.

Conclusion-The low yield of positive results and lack of evidence of impact on management indicate that DMSA scanning, with all the implications of isotope exposure, intravenous injection, staff time, psychological trauma, and expense, could be omitted in children over 1 year with first simple UTI not sufficiently ill to be admitted to hospital. The low rate of detection of UTI in primary care in infants may represent under diagnosis. (Arch Dis Child 2001;84:324-327)
\end{abstract}

Keywords: urinary tract infection; inpatients; outpatients; DMSA scan; guidelines

University Hospital of Wales

P V Deshpande

KRUF Children's Kidney Centre for Wales, University of Wales College of

Medicine, Heath Park, Cardiff CF14 4XN, UK $\mathrm{K}$ Verrier Jones

Correspondence to:

Dr Verrier Jones

verrier-jones@cf.ac.uk

Accepted 30 November 2000

Urinary tract infection (UTI) is one of the commonest bacterial infections of childhood, accounting for around 5\% of febrile illnesses. ${ }^{1}$ Over 30 years ago imaging studies carried out on children with UTI showed that renal scarring was present in $10-25 \%$ and vesicoureteric reflux (VUR) was present in $30 \%{ }^{2}$ Renal scarring was worse in children who developed a first infection at a young age, ${ }^{3}$ had recurrent UTI, had VUR, and where there was a delay in diagnosis and treatment. ${ }^{4}$ Because of the high incidence of anomalies in these series and the risk of progression of renal damage, recommendations were made for routine imaging of children following UTI.

In 1991 a working group of the Royal College of Physicians published guidelines on the diagnosis and management of UTI in children. ${ }^{5}$ These guidelines recommend that all children should have an ultrasound scan of the urinary tract with an abdominal $x$ ray. In addition, the working group recommended that all children less than 7 years should have a dimercaptosuccinic acid (DMSA) scan and all children less than 1 year should have a micturating cystogram (MCUG). ${ }^{5}$ These recommendations were based on observations from a number of studies showing the high incidence of VUR and renal scarring, and the belief that renal scarring could be prevented by prophylactic antibiotic therapy or reimplantation of the ureter. ${ }^{67}$ At that time guidelines were not subjected to the same rigorous care in preparation that would be expected now, a decade later. ${ }^{8}$ In particular, the value of invasive imaging tests was not supported by evidence that knowledge of their presence altered management or prognosis. The huge cost of carrying out these tests in this common condition and the trauma and radiation to children were barely considered.

The guidelines were a valuable first step in rationalisation of the diagnosis and management of early childhood UTI and have encouraged some uniformity of practice which has brought about some rationalisation of management. However, in clinical practice since the guidelines were published there has been more emphasis on the imaging tests recommended after full recovery from the infection than on the diagnosis and treatment of infection. In early childhood, particularly in children under 2 years, the diagnostic process is fraught with difficulty and confusion because of problems with urine collection, contamination of samples, and uncertainty about laboratory criteria for diagnosis. ${ }^{9}$

The aims of the study were to assess the extent to which local practice followed the guidelines published by the Royal College of Physicians in 1991 and to evaluate the outcome
Table 1 Ages of children treated for urinary tract infection at home (outpatients) and in hospital (inpatients)

\begin{tabular}{|c|c|c|c|c|c|c|}
\hline \multirow[b]{2}{*}{ Age (y) } & \multicolumn{2}{|c|}{ Outpatients } & \multicolumn{2}{|c|}{ Inpatients } & \multicolumn{2}{|c|}{ Total } \\
\hline & No. & $\%$ & No. & $\%$ & No. & $\%$ \\
\hline 0 to $<1$ & 13 & 8 & 43 & 26 & 56 & 34 \\
\hline 1 to $<7$ & 69 & 42 & 20 & 12 & 89 & 54 \\
\hline $7-12$ & 13 & 8 & 6 & 4 & 19 & 12 \\
\hline Total & 95 & 58 & 69 & 42 & 164 & 100 \\
\hline
\end{tabular}


Table 2 Imaging investigations carried out as a result of local guidelines according to age in children treated for urinary tract infection at home (outpatients) and in hospital (inpatients)

\begin{tabular}{|c|c|c|c|c|c|c|c|c|c|c|c|}
\hline \multirow[b]{2}{*}{ Age (y) } & \multirow[b]{2}{*}{ Investigation } & \multicolumn{2}{|c|}{ Outpatients } & \multicolumn{2}{|c|}{ Inpatients } & \multicolumn{2}{|l|}{ Total } & \multirow[b]{2}{*}{$T F$} & \multirow[b]{2}{*}{$N R$} & \multirow[b]{2}{*}{$D N A$} & \multirow[b]{2}{*}{$P D$} \\
\hline & & No. & $\%$ & No. & $\%$ & No. & $\%$ & & & & \\
\hline \multirow{3}{*}{0 to $<1$} & Ultrasound & $13 / 13$ & 100 & $43 / 43$ & 100 & $56 / 56$ & 100 & 0 & 0 & 0 & 0 \\
\hline & DMSA & $10 / 13$ & 77 & $42 / 43$ & 98 & $52 / 56$ & 93 & 1 & 1 & 1 & 1 \\
\hline & MCUG & $11 / 13$ & 85 & $39 / 43$ & 91 & $50 / 56$ & 89 & 1 & 0 & 4 & 1 \\
\hline \multirow[t]{2}{*}{1 to $<7$} & Ultrasound & $69 / 69$ & 100 & $19 / 20$ & 95 & $88 / 89$ & 99 & 0 & 0 & 1 & 0 \\
\hline & DMSA & $54 / 69$ & 78 & $18 / 20$ & 90 & $72 / 89$ & 81 & 3 & 11 & 2 & 1 \\
\hline $7-12$ & Ultrasound & $13 / 13$ & 100 & $6 / 6$ & 100 & $19 / 19$ & 100 & 0 & 0 & 0 & 0 \\
\hline \multirow[t]{3}{*}{ Total } & Ultrasound & $95 / 95$ & 100 & $68 / 69$ & 99 & $163 / 164$ & 99 & 0 & 0 & 1 & 0 \\
\hline & DMSA & $64 / 82$ & 78 & $60 / 63$ & 95 & $124 / 145$ & 86 & 4 & 12 & 3 & 2 \\
\hline & MCUG & $11 / 13$ & 85 & $39 / 43$ & 91 & $50 / 56$ & 89 & 1 & 0 & 4 & 1 \\
\hline
\end{tabular}

TF, technical failure; NR, not requested; DNA, did not attend; PD, parents declined.

of imaging tests carried out as a result of the guidelines in inpatients and outpatients. ${ }^{5}$

\section{Patients and methods}

PATIENTS

The children were all seen at the University Hospital of Wales or the Cardiff Royal Infirmary between 1 February 1997 and 31 January 1998, either as inpatients or as outpatients. The outpatients had been diagnosed and managed at home by their general practitioners and referred following treatment for imaging investigations. A UTI was defined as a urine culture showing a pure growth of more than $10^{5}$ colony forming units $/ \mathrm{ml}$ of urine collected by bag, clean catch, or mid stream sample.

EXCLUSIONS

All children without clear laboratory evidence of UTI as defined above were excluded from the study. In addition any child who had been previously investigated for UTI, any child investigated because of renal or urinary tract anomalies detected as a result of ultrasound screening before or after birth, and any child with a major congenital anomaly was excluded.

GUIDELINES

The local guidelines, based on recommendations of the Royal College of Physicians, were available in a departmental handbook updated annually, kept on all children's wards and given to all medical staff. The guidelines recommended that all children should have an ultrasound scan. In addition children under 7 years should have a Tc99m DMSA isotope scan carried out at least three months after treatment of

Table 3 Prevalence of abnormalities detected by ultrasound, ${ }^{99 m}$ Tc DMSA scanning, and MCUG in children treated for UTI at home (outpatients) and in hospital (inpatients)

\begin{tabular}{|c|c|c|c|c|c|c|c|}
\hline \multirow[b]{2}{*}{ Age (y) } & \multirow[b]{2}{*}{ Investigation } & \multicolumn{2}{|c|}{ Outpatients } & \multicolumn{2}{|c|}{ Inpatients } & \multicolumn{2}{|l|}{ Total } \\
\hline & & No. & $\%$ & No. & $\%$ & No. & $\%$ \\
\hline \multirow[t]{3}{*}{0 to $<1$} & Ultrasound & $0 / 13$ & 0 & $7 / 43$ & 16 & $7 / 56$ & 13 \\
\hline & DMSA & $0 / 10$ & 0 & $7 / 42$ & 17 & $7 / 52$ & 14 \\
\hline & MCUG & $3 / 11$ & 27 & $14 / 39$ & 36 & $17 / 50$ & 34 \\
\hline \multirow[t]{2}{*}{1 to $<7$} & Ultrasound & $2 / 69$ & 3 & $5 / 19$ & 26 & $7 / 88$ & 8 \\
\hline & DMSA & $1 / 54$ & 2 & $6 / 18$ & 33 & $7 / 72$ & 10 \\
\hline $7-12$ & Ultrasound & $1 / 13$ & 8 & $1 / 6$ & 17 & $2 / 19$ & 11 \\
\hline \multirow[t]{3}{*}{ Total } & Ultrasound & $3 / 95$ & 3 & $13 / 68$ & 19 & $16 / 163$ & 10 \\
\hline & DMSA & $1 / 64$ & 1 & $13 / 60$ & 22 & $14 / 127$ & 11 \\
\hline & MCUG & $3 / 11$ & 27 & $14 / 39$ & 36 & $17 / 50$ & 34 \\
\hline
\end{tabular}

the UTI to show renal scarring. Micturating cystography to detect VUR was recommended for all children under 1 year.

STATISTICAL ANALYSIS

This was carried out using the $\chi^{2}$ test with Fisher's two tailed values.

\section{Results}

After exclusion of cases not meeting the criteria, 164 sets of case notes (51 boys, 113 girls) were examined (table 1). Table 2 shows results of imaging investigations for inpatients and outpatients. Table 3 shows the abnormalities detected on imaging, carried out in accordance with the guidelines. In addition, four of 20 children (20\%) aged 1 to 6 years had VUR on MCUG and two of six children (33\%) over 7 years (one of three inpatients and one of three outpatients) had scarring on DMSA scans, although these tests were additional to the minimum recommended in the guidelines. A smaller proportion of infants were diagnosed at home than of other age groups $(p<0.01)$. The prevalence of renal scarring on DMSA scan in children over 1 year was lower in children diagnosed and treated at home compared to children admitted to hospital $(p<0.01)$. The time between diagnosis of UTI and DMSA scan was 4.3 months and 3.6 months, respectively, for children under 1 and 1-6 years admitted to hospital, and 5.0 months and 4.7 months for children managed at home. The time interval between diagnosis of UTI and DMSA scan for children with normal scans was 4.4 months and 3.5 months, and for abnormal scans was 4.0 months and 4.0 months, for children under 1 year and children aged 1-6 years, respectively. Children with VUR or renal scarring were advised to continue prophylaxis for one to two years, while children with normal scans were advised to stop prophylaxis.

\section{Discussion}

The guidelines on management of childhood UTI were published in 1991 as a consensus statement following a one day meeting of clinicians interested in UTI in childhood. ${ }^{5}$ There was general agreement that children with UTI benefited from imaging investigations, although evidence that these tests alter the immediate or long term outcome is weak. ${ }^{10}$ The 
reason stated for benefit from tests was that "a significant number of important abnormalities will be detected in both sexes". However, no evidence was provided in support of the hypothesis that doing the tests altered the outcome, other than providing the opportunity to discuss the abnormality and anticipate complications. There was significant disagreement between participants about the extent to which young children should be investigated and the most appropriate tests to show renal scarring at different ages. There was agreement that all children should have an ultrasound scan following UTI and that the youngest children should have the most thorough investigation, but there was no clear consensus on the extent of other investigations. The working group made a set of recommendations, as an interim measure, for the minimum imaging investigations in straightforward cases, recognising that further research would be required to enable definitive guidelines to be established.

Ultrasound was recommended for all children, while DMSA scintigraphy was recommended in addition for all children under 7 years; micturating cystography was recommended in addition for infants under 12 months. Although the guidelines stated that the need for these tests should be reviewed when results of further research became available, there has been little published evidence challenging the guidelines which are now widely used by paediatricians throughout the UK. In contrast to practice in many other countries, in the UK little attempt has been made to distinguish clinically between lower tract infection and acute pyelonephritis beyond the evidence obtained by history and examination, and there was no attempt to make this potentially important distinction in the guidelines. Consequently many children with trivial illnesses, diagnosed and treated at home by general practitioners, are now routinely subjected to DMSA scans in addition to ultrasound, although there is some doubt about the value of this. Many children under 7 years also receive long term low dose prophylaxis while awaiting imaging investigations without clear evidence that this is effective in preventing renal scar formation. Some parents now question the wisdom of long term prophylaxis for several months following a trivial illness and compliance has been shown to be poor. ${ }^{11}$

These imaging tests are not done to aid the diagnosis or treatment of the acute illness but are carried out later when the child has recovered fully and are therefore a form of screening carried out in a high risk population. These children are perceived to be at risk of having underlying anomalies and of acquiring renal damage. However, the World Health Organisation's criteria for a successful screening programme are not met. ${ }^{12}$ The detection of anomalies in a third of cases is not sufficient justification for carrying out these tests. There should be some evidence that the new knowledge derived from the investigation can alter management and outcome to justify screening.
Although much is known about the natural history of UTI, VUR, and renal scarring, little evidence exists that the natural history can be altered. Scars remain static or progress in a proportion of children, even when given excellent care and supervision. ${ }^{13}{ }^{14}$ This may be in part because UTI is easily overlooked in infants at the age when there is the greatest risk of renal scarring. ${ }^{9}$ Much of the preventable damage may have occurred prior to entry into the two largest controlled studies. ${ }^{13} 14$

Only $23 \%$ of infants with UTI included in our study were diagnosed in primary care, compared with $77 \%$ managed in hospital; in older children the ratio was reversed. In a pilot study, van der Voort et al showed that children with UTI had visited their general practitioners twice as often as controls prior to diagnosis of the first UTI. ${ }^{15}$ This suggests that UTI may have been overlooked, thus predisposing to renal scar formation.

We have used a simple process, hospital admission, to identify children who were likely to have had severe or systemic illness and to distinguish them from those who were treated at home and who were more likely to have had a trivial illness. Of the children under 1 year, $13 / 56(23 \%)$ were diagnosed in primary care compared with $77 \%$ diagnosed in hospital. This may reflect a higher rate of upper tract infection in this subgroup or the difficulty general practitioners have in establishing the diagnosis of UTI in infancy. ${ }^{9}$ The incidence of UTI is highest and the risk of renal damage is greatest in the first year of life. ${ }^{416}$ The recommendations in the guidelines that infants should have ultrasound, DMSA, and MCUG were generally accepted by members of the working group. In our study renal scarring was shown in $14 \%$ of infants, and $17 \%$ of those infants treated in hospital. Although no scars were shown in infants diagnosed at home, the numbers are too small for statistical significance or comment. There is a possibility that there may have been some false positive diagnoses of UTI. ${ }^{17}$

In children aged 1-6 years, only one child $(2 \%)$ of the 54 treated at home showed any sign of renal scarring compared with $6 / 18(33 \%)$ of those admitted to hospital. This difference is both statistically and clinically significant. The time from UTI to DMSA scan was slightly shorter in children managed in hospital. This difference could bias the results in favour of scars in the group managed in hospital but would not affect the conclusions of this study that children over 12 months insufficiently ill to require admission do not benefit from DMSA scans.

DMSA scans are invasive tests that involve an intravenous injection, several hours in hospital, radiation to the child and the gonads, and in some cases, sedation or restraint. There is a risk of psychological trauma to the child and the investigation is also stressful to parents. ${ }^{18}$ Parents may lose time from work and there are financial costs of travelling with young children. In our study the DMSA was abandoned in four children because of technical failure and in two cases the parents did not 
wish their children to undergo the investigation. In 12 cases it was not requested by the medical staff, perhaps because they did not feel that it was indicated. The test costs around $£ 120$, with additional costs in smaller children who are often admitted as day cases for sedation. Stark has questioned the economic value of routine imaging of the urinary tract in children $^{19}$ and Chambers has questioned the process from the child's perspective. ${ }^{20}$ On the basis of $£ 120$ per DMSA scan, the cost of detecting one scar in this series was $£ 1100$ pounds. If selected at risk groups had been targeted and fewer scans had been carried out, the cost could have been reduced. The cost of detecting each scar in infants admitted to hospital with UTI was $£ 720$; in children over 7 years with clinical evidence of acute pyelonephritis in this series, the cost was $£ 360$ per scar. In contrast, the cost of detecting one scar in children managed as outpatients was $£ 7680$. Perhaps this money would be better spent on resources in primary care to improve the diagnosis and treatment of UTI in infants and toddlers. ${ }^{9}$

This small study indicates that there are reasonable grounds to question the benefit of carrying out a DMSA scan on every child under 7 years with a proven UTI based on risks and benefits to the patient and on the cost to the NHS. The clinician should be free to recommend a DMSA scan in a child with a history suggestive of acute pyelonephritis, or in cases where there is a history of delay in treatment or frequent recurrences of UTI. The conclusion from this study is that routine use of DMSA scans in children over 1 year with a straightforward simple infection is unhelpful and unnecessary.

1 Hoberman A, Chao H-P, Keller DM, et al. Prevalence of urinary tract infection in febrile infants. $f$ Pediatr 1993;123:17-23.
2 Hodson CJ. The kidneys in urinary tract infection. Proc $R$ Soc Med 1966;59:416.

3 Berg UB, Johansson SB. Age as a main determinant of renal functional damage in urinary tract infection. Arch Dis Child 1983;58:963-9.

4 Smellie JM, Ransley PG, Normand ICS, et al. Development of new renal scars; a collaborative study. BMF 1985;290: 1957-60.

5 Report of a Working Group of the Research Unit Royal College of Physicians. Guidelines for the management of acute urinary tract infection in childhood. $\mathcal{F} R$ Coll Physicians Lond 1991;25:36-42.

6 Smellie JM, Normand C. Urinary tract infection and reflux nephropathy in children. Med Intern 1991;87:3610-17.

7 Bellinger MF, Duckett JW. Vesicoureteric reflux: a comparison of non-surgical and surgical management. Contrib Nephrol 1984;39:81-93.

8 Quality of Practice Committee Royal College of Paediatrics and Child Health. Standards for development of clinical guidelines in paediatrics and child health: role of the Royal College of Paediatrics and Child Health. London: Royal College of Paediatrics and Child Health, 1998.

9 van der Voort J, Edwards A, Roberts R, Verrier Jones K. The struggle to diagnose UTI in children under two in primary care. Fam Pract 1997; 14:44-8.

10 Larcombe J. Urinary tract infection in children: clinical evidence. BM7 1999;319:1173-5.

11 Smyth AR, Judd BA. Compliance with antibiotic prophylaxis in urinary tract infection. Arch Dis Child 1993;68:2356.

12 Verrier Jones K. Screening after urinary tract infection in childhood. Arch Dis Child 2000;28:338.

13 Birmingham Reflux Study Group. Prospective trial of operative versus non-operative treatment of severe vesicoureteric reflux in children: five years' observation. BMF 1987;295:237-41.

14 Smellie JM, Tamminen-Mobius T, Olbing H, et al. Five-year study of medical or surgical treatment in children with severe reflux: radiological renal findings. Pediatr Nephrol 1992;6:223-30.

15 van der Voort J, Verrier Jones K, Edwards A. Can renal scarring be prevented by earlier diagnosis of urinary tract infection in infancy? Kidney Int 1997;52:1130.

16 Winberg J, Bergstrom T, Jacobsson B. Morbidity, age and sex distribution, recurrences and renal scarring in symptomatic urinary tract infection in childhood. Kidney Int 1975; 8:S101-S106.

17 Liaw L, Nayar D, Pedler S, Coulthard M. Home collection of urine for culture from infants by three methods: survey of parents preferences and bacterial contamination rates. BMF 2000;320:1312-13.

18 Phillips D, Watson AR, Collier J. Distress and radiological investigations of the urinary tract in children. Eur $\mathcal{F}$ Pediatr 1996;155:684-7.

19 Stark H. Urinary tract infections in girls: the costeffectiveness of currently recommended investigative routines. Pediatr Nephrol 1997;11:174-7.

20 Chambers T. Commentary on article by H Stark. An essay on the consequences of childhood urinary tract infection. Pediatr Nephrol 1997;11:178-9. 